\title{
Isolation, molecular and phylogenetic identification of microorganisms from Kombucha solution and evaluation of their viability using flow cytometery
}

\author{
Reyhaneh JAFARI ${ }^{1}$, Nafiseh Sadat NAGHAVI ${ }^{1 *}$, Kianoush KHOSRAVI-DARANI ${ }^{2 *}$ (D), Monir DOUDI ${ }^{1}$, \\ Kahin SHAHANIPOUR ${ }^{3}$
}

\begin{abstract}
Kombucha can be produced by coculture of a consortium of bacteria and fungi in a sweetened black tea. Determining the type of microbial population as well as their survival is important because they lead to beneficial and sensory properties of the product. In this study, microorganisms were isolated from kombucha and identified using culture and molecular methods and vacuum dried. Also total phenol content, and antioxidant properties of beverage were characterized. Microorganism viability and sensory evaluation was conducted by flow cytometery and a 5-point hedonic test before and after drying. 8-isolated species were recorded in NCBI. Lactobacillus and Kumagataeibacter and Weissella spp. were the bacterial species, while Starmella bacillaris and Hanseniaspora uvarum were the 2-yeast species identified in kombucha beverage. Microbial count increased from 3.59\% on the start day to $96.6 \%$ on day 14 . The results of sensory evaluation show that, in general, the produced kombucha drink was accepted by the evaluators. The highest score for overall acceptance on day 14 was equal to 4.6. Starmella bacillaris and Hanseniaspora uvarum were first isolated from kombucha with enhanced antioxidant properties. These strains, together with yeast are recommended for use in beverage fermentation processes to production of bioactive compounds.
\end{abstract}

Keywords: Kombucha beverage; antioxidant capacity; viability; flow cytometery.

Practical Application: Determining the type of microbial consortium and survival in the beverage. as the microbial population which can be useful for bioavailability of chemical compounds and, consequently, the sensory properties and overall acceptance

\section{Introduction}

In last decade, nondairy herbal drinks have attracted the attention of consumers and researchers due to their beneficial effects on health resulted from their content of polyphenols (Annuzzi et al., 2014). Kombucha is a fermented probiotic and carbonated beverage produced by co-cultures of bacteria and osmophilic yeasts in sweeten tea (Amarasinghe et al., 2018; Yikmis \& Tuggum, 2019; Gaggia et al., 2018; Zhao et al., 2018; Kozyrovska et al., 2012). Fermentation of kombucha includes production of alcohol, lactic and acetic acid (VillarrealSoto et al., 2018). in the first days of fermentation, glucose and fructose will be produced from sucrose by the microbial invertase enzyme. Then yeast convert glucose to $\mathrm{CO}_{2}$ and ethanol (Sreeramulu et al., 2000; Loncar et al., 2006). Acetobacteria play their role by converting ethanol to acetic acid. Ethanol, acetic acid, and gluconic acid are the main products kombucha tea fermentation (Velićanski et al., 2014; Vazquez-Cabral et al., 2014).

Up to now, many compounds such as carbonic, acetic, gluconic, folic, lactic, glucuronic, citric oxalic, and malic acid, as well as vitamins $B$ group $\left(B_{1}, B_{2}, B_{3}, B_{6}\right.$ and $\left.B_{12}\right)$ and vitamin $\mathrm{C}$, has been identified in kombucha beverage (Catechins, tiaflavin, Flavonol, etc.). Metabolic enzymes including invertase, amylase and other oxidative enzymes are also extracted from kombucha drink.
The health beneficial impacts of kombucha has reported for treatment of several diseases including diabetes, the risk of heart disease (by reducing cholesterol and blood pressure), liver detoxification (by binding glucuronic acid to toxins), losing weight, relieving arthritis and stimulating the immune system, as well as preventing cancer (Dufresne \& Farnworth, 2000; Srihari \& Satyanarayana, 2012; Malbasa et al., 2014; Gamboa-Gómez et al., 2016, Jayabalan et al., 2007). Also acetic acid content of kombucha resulted in antimicrobial inhibitory impact (against Bacillus cereus, Helicobacter pylori, Shigella sonei, Staphylococcus aureus, Escherichia coli, Salmonella interatidicus) and antifungal properties of kombucha (Battikh et al., 2013; Reiss, 1994). Fermentation of kombucha leads to release of polyphenols, e.g. catechins, with high antioxidant properties (Srihari \& Satyanarayana, 2012; Jayabalan et al., 2007). Also, kombucha is rich in antioxidants such as vitamins $\mathrm{C}$ and E, beta-carotene and other carotenoids (Malbasa et al., 2014; Gamboa-Gómez et al., 2016). Kombucha is not only a probiotic but also act as a prebiotic (due to its microcellulose layer) to help the growth of beneficial microbes in the gut (Kozyrovska et al., 2012; Dufresne \& Farnworth, 2000).

Determining the type of microbial population as well as their viability in the beverage is very important, as the microbial 
population in kombucha affects the bioavailability of chemical compounds and, consequently, the sensory properties and overall acceptance of the product. In the present study, isolation and purification of microorganisms from kombucha solution was performed using culture method and molecular identification of microorganisms was performed using PCR and viability of them performed by flow cytometery.

\section{Materials and methods}

\subsection{Preparation of kombucha beverage}

Preparation of kombucha beverage was described elsewhere in details (Jafari et al., 2020).

\subsection{Isolation and purification of microorganisms in kombucha solution}

Man Rogosa Sharpe Agar (MRS) containing 0.1\% cyclohexamide, Glucose Yeast Chalk Agar (GYCA) containing $3 \%$ ethanol (95\%), and potato dextrose agar (PDA) containing $100 \mathrm{mg} / \mathrm{L}$ Chloramphenicol were respectively used for isolation and purification of lactic acid bacteria (LAB), acetic acid bacteria, and fungi (Goh et al., 2012; Gaggia et al., 2018). All media obtained from Sigma-Aldrich, USA. Cultured yeasts were incubated at $25-28^{\circ} \mathrm{C}$ for $72 \mathrm{~h}$ and cultured bacterial were incubated at $30^{\circ} \mathrm{C}$ for $48 \mathrm{~h}$. The isolated bacteria were identified for initial diagnosis using macroscopic observations of colonies and microscopic observations after gram staining as well as biochemical tests. The microscopic appearance of fungi was evaluated by light microscopy (Nikon, Japan).

\subsection{Molecular identification of microorganisms in kombucha solution using PCR}

\section{Molecular identification of bacteria}

DNA extraction kit (RIBO-prep, nucleic acid extraction kit, made in Russia) was used to extract DNA from bacterial and fungal colonies. For bacterial samples, the 16S rRNA gene was amplified using DG74 (5'AGGAGGTGATCCAACCGCA3') and Rw01 (5'ACTGGAGGAAGGTGGGG13) primers (Coton et al., 2017; Marsh et al., 2014). The thermal protocol comprises initial denaturation at $95^{\circ} \mathrm{C}$ for 5 minutes, followed by 30 cycles including denaturation at $95^{\circ} \mathrm{C}$ for 30 seconds, primer annealing at $58^{\circ} \mathrm{C}$ for 30 seconds, and extension at $72^{\circ} \mathrm{C}$ for 1 minute. This process was completed with the final extension at $72{ }^{\circ} \mathrm{C}$ for 5 minutes.

\section{Molecular identification of yeasts}

For yeast isolates, the 18S rRNA gene was amplified using ITS1: (5'TCCTCCGCTTATTGATATGC3') and ITS4 (5'TCCGTAGGTGAACCTGCGG 3') primers (Marsh et al., 2014; Matei et al., 2018). The thermal protocol was included an initial denaturation at $94^{\circ} \mathrm{C}$ for 5 minutes, followed by 30 cycles including denaturation at $94^{\circ} \mathrm{C}$ for 30 seconds, primer annealing at $55^{\circ} \mathrm{C}$ for 1 minutes, and extension at $72^{\circ} \mathrm{C}$ for 45 seconds. The process was ended by a final extension at $72{ }^{\circ} \mathrm{C}$ for 5 minutes.

\subsection{Sequencing of PCR products}

In order to definitively identify the microorganisms, present in kombucha solution, PCR products were transferred to Gene Technologies Company (Tehran, Iran) for sequencing by Sanger method (Sanger et al., 1977). Then, the determined sequences were blasted with other sequences in the gene bank and the isolated species were identified with the highest similarity (98-100\%) and the lowest E-value in terms of genus and species. Phylogenetic tree mapping for the studied species was performed using MEGA7 software. After editing the sequences and removing the heterogeneous regions, the phylogenetic tree was drawn using the neighborhood-connection method. In this method, the Q matrix is used, which includes all branches and selects the least value that expresses the high similarity between the two branches and uses it in a branch of the phylogenetic tree. Bootstrapping values were obtained after 1000 re-testing.

\subsection{Phylogeny and determining the evolutionary pathway}

Phylogenetic tree mapping for the studied species was performed using MEGA7 software. After editing the sequences and removing the heterogeneous regions, the phylogenetic tree was drawn using the neighborhood-connection method. In this method, the $\mathrm{Q}$ matrix is used, which includes all the branches and selects the lowest value that expresses the high similarity between the two branches and uses it in a branch of the phylogenetic tree. Bootstrapping values were obtained after 1000 re-testing.

\subsection{Drying the kombucha solution}

Drying the kombucha solution was described in details elsewhere (Jafari et al., 2020).

\section{7 pH monitoring}

Evaluation of $\mathrm{pH}$ of kombucha before drying (A) and after drying (B) on different days of fermentation was described in details elsewhere (Jafari et al., 2020).

\subsection{Determination of the total phenol content and anti- oxidant activity of kombucha}

The total phenol content and antioxidant activity of the kombucha before and after drying was measured by Folin-Ciocalteau method (Singleton et al., 1999), and radical 1,1-diphenyl 2-picrylhydrazyl absorption and cupric ion reducing antioxidant capacity methods (Jayabalan et al., 2007; Yikmis \& Tuggum, 2019), respectively.

\subsection{Evaluation of survival power of microorganisms using flow cytometery}

The viability of microorganisms in two groups A (kombucha solution before drying) and B (kombucha solution after drying), in the intervals before the start of fermentation (day zero), the seventh day, the tenth day and the fourteenth day, was measured to the general form and separately for fungi and bacteria using flow cytometry. For this purpose, rhodamine 123 dye was used to stain the cells. To prepare the dye solution, $5 \mathrm{mg}$ of the dye was dissolved in $5 \mathrm{~mL}$ of 
ethanol. The prepared rhodamine 123 solution was stored at $-20{ }^{\circ} \mathrm{C}$ in a dark place. For each use, the dye was diluted in PBS buffer in a ratio of 1 to 100 . To check the viability of microorganisms by flow cytometery, $400 \mu \mathrm{L}$ of the dye was added to $100 \mu \mathrm{L}$ of kombucha solution, and after placing at room temperature for 10 minutes, the amount of viability was measured in a flow cytometer (Flow rate, BD FACS Callibur, USA). The excitation or absorption wavelength of rhodamine 123 dye is 507 to $560 \mathrm{~nm}$ and the emission wavelength of this dye is 529 to $580 \mathrm{~nm}$ (Gholami \& Etemadifar, 2013).

\subsection{Sensory evaluation of kombucha solution}

Sensory evaluation of kombucha solution before and after drying was performed on days $0,7,10$ and 14 of fermentation by 15 evaluators. Sensory evaluation was performed based on 5 -point hedonic test. The test scale was $(5=$ very good, $4=$ good, $3=$ moderate, $2=$ bad and $1=$ very bad). Sensory evaluation parameters included taste, aroma, color, appearance, and overall acceptance of the product (Shahbazi et al., 2018).

\section{Results}

\subsection{The results of isolation and identification of microorganisms}

Isolation, purification and apparent identification of microorganisms in kombucha solution was performed using the culture method in the relevant microbiological culture medium. Figure 1 shows a microscopic image of some of the isolated samples.

\subsection{The results of molecular amplification (PCR) and sequencing of isolated microorganisms}

PCR was performed to exact identification of the samples isolated from kombucha solution. The electrophoresis results of bacterial PCR isolates are shown in Figures 2A and the results of electrophoresis of fungal isolated PCR products are shown in Figure 2B. PCR products were sequenced using sanger method and compared with existing sequences by referring to the gene bank. The phylogenetic tree for the isolated specimens is shown in Appendix A. Finally, the sequences of genes for bacterial and fungal strains that were more molecularly and phylogenetically similar to the bacteria and fungi were recorded in the NCBI database with the appended numbers listed in Table 1.

\subsection{Evaluation of pH during kombucha fermentation}

The results of $\mathrm{pH}$ measurements during kombucha fermentation are presented in Figure 3.

\subsection{The results of total phenolic content, DPPH free radical scavenging activity, and CUPRAC method assay}

Antioxidant activity and total phenol content in two kombucha solutions were measured before drying (A) and after drying (B). Based on the results of the total phenol content in kombucha solution, the amount of 245.31-285.44 mg of gallic acid in $1 \mathrm{~mL}$ of kombucha solution was detected during 14 days of fermentation. The percentage of free radical scavenging of $\mathrm{DPPH}$ and the antioxidant capacity of the samples increased by CUPRAC method on average during the fermentation time. The percentage of DPPH radical inhibition at the end of the fermentation period in the two samples was in the range of 36.78-89.32. Antioxidant capacity of samples A and B had a range of $11.52-224.86$ equivalents to trolox $\mathrm{nmol} / \mathrm{mL}$ of kombucha solution. Table 2 presents the results from total phenol content, DPPH free radical scavenging activity, and CUPRAC method assay.

\subsection{Results of microorganism viability using flow cytometery}

Microorganism viability was measured in kombucha solutions before drying (group A) and after drying (group B), day zero (before fermentation), day 7, day 10 and day 14, using flow cytometery method. According to the results that are shown in Figures 4, the overall viability of microorganisms has increased on average during the fermentation time, as in group A, it showed an altitude from $3.59 \%$ on day zero fermentation to $96.6 \%$ on day 14 . In group B it increased from $2.58 \%$ on day zero fermentation to $90.5 \%$ on day 14 . viability of microorganisms by fungi and bacteria using flow cytometry in kombucha solution before and after drying are shown in Table 3. According to the results of determining the viability of microorganisms by bacteria and fungi, in group $A$ and $B$ the viability of fungi on days 0,7 and 10 was much higher than bacteria. But on day 14, the viability of bacteria is almost double that of their survival on day 10 . On the other hand, survival of bacterial was higher than fungi on day 14 .

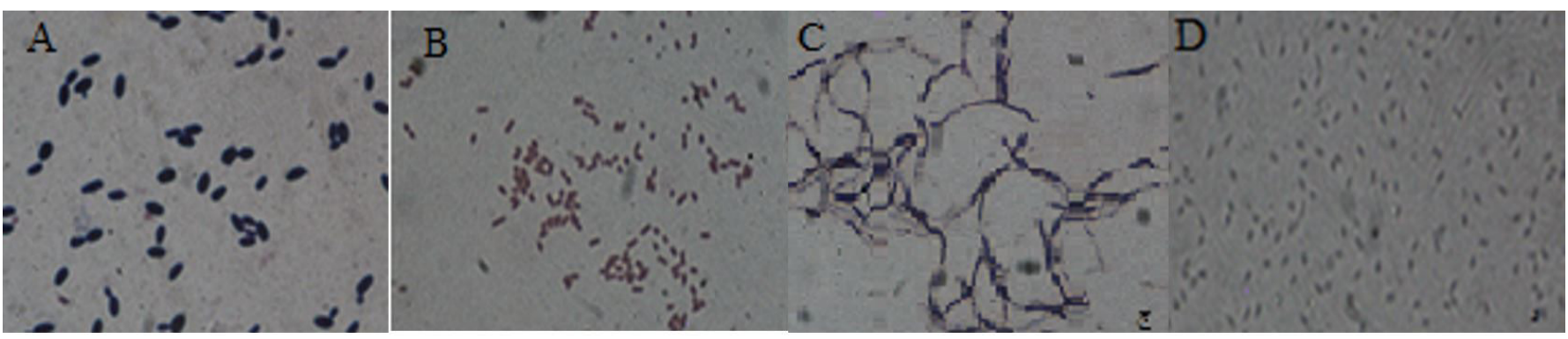

Figure 1. Results of gram staining of isolated samples: (A) ovary yeast cells; (B) Gram-positive bacterium cocci; (C) Gram-positive bacilli with strep to arrangement; (D) Gram-positive coccobacillus with single arrangement. 


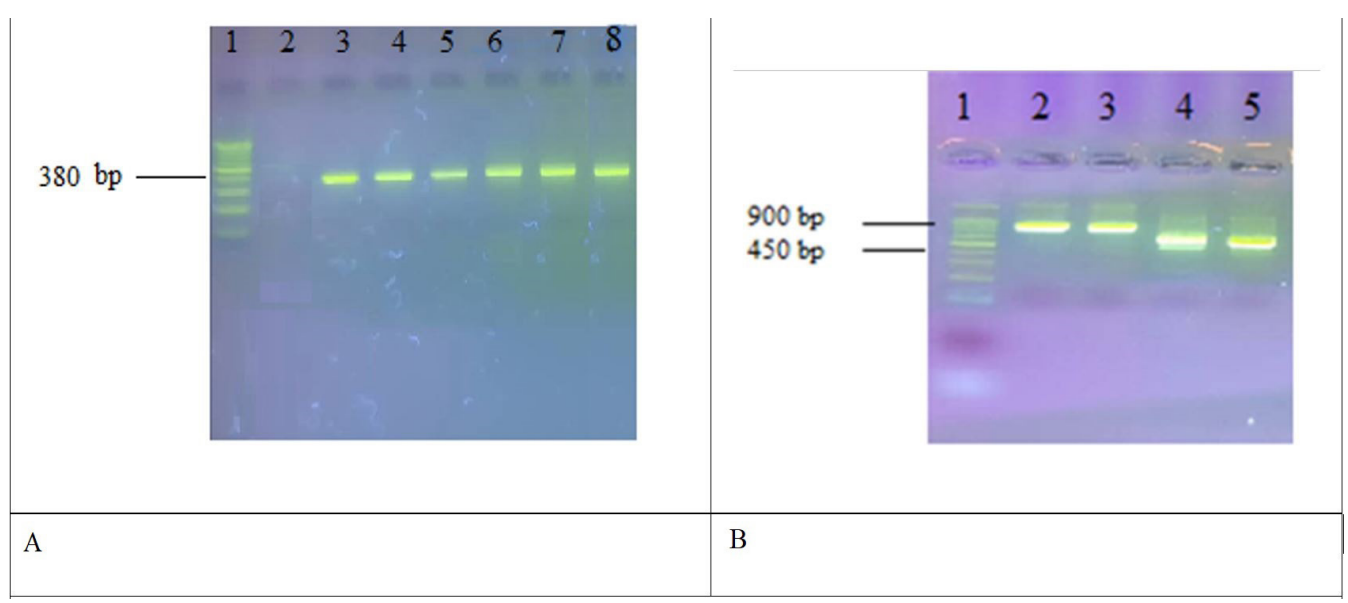

Figure 2. Results of electrophoresis of PCR products: (A): Samples from left to right 1: DNA Ladder (100 bp), 2: negative Samples, 3-8: isolated bacterial Samples; (B) Samples from left to right 1: DNA Ladder (100 bp) and 2-5: fungal samples isolated from kombucha solution.

Table 1. The fungal and bacterial strains isolated from kombucha solution.

\begin{tabular}{ccc}
\hline Numbers & Isolated sample (similarity percentage) & Accession number \\
\hline 1 & Kumagataeibacter xilinus kombu NKJ1 (99.37\%) & MT919949 \\
2 & Kumagataeibacter saccharivorans kombu NKJ2 (99.37\%) & MT919897 \\
3 & Starmella bacillaris kombu NKJ3 (100\%) & MT919949 \\
4 & Weissella confusa kombu NKJ4 (98\%) & MT920108 \\
5 & Hanseniaspora uvarum kombu NKJ5 (99.43\%) & MT921612 \\
6 & Lactobacillus rhamnosus kombu NKJ6 (99.48\%) & MT921616 \\
7 & Lactobacillus acidophilus kombu NKJ7 (99.76\%) & MT921617 \\
8 & Lactobacillus acidophilus kombu NKJ8 (99.76\%) & MT921623 \\
\hline
\end{tabular}

Table 2. Total phenol content (TPC); DPPH (1,1-diphenyl 2-picrylhydrazyl), and cupric ion reducing antioxidant capacity (CUPRAC) analysis in kombucha solutions before drying (group A) and after drying (group B) during the fermentation process.

\begin{tabular}{ccccc}
\hline Samples & Days & TPC mg GAL/mL & DPPH $(\%$ inhibition $)$ & CUPRAC $\mathrm{nm}$ trolox/mL \\
\hline A & 7 & $262.31 \pm 0.98$ & $70.47 \pm 0.01$ & $50.00 \pm 0.99$ \\
& 14 & $278.32 \pm 0.49$ & $89.32 \pm 0.66$ & $224.86 \pm 1.85$ \\
B & 7 & $0.026^{*}$ & $0.002^{*}$ & $<0.001^{*}$ \\
& 14 & $250.41 \pm 0.77$ & $45.08 \pm 0.01$ & $13.00 \pm 0 / 50$ \\
& $p$-value $\$$ & $285.44 \pm 1.08$ & $85.43 \pm 0.93$ & $219.72 \pm 9.85$ \\
\hline
\end{tabular}

The results are presented as mean \pm standard deviation (SD). \$ Using the Wilcoxon signed rank test. ${ }^{*}$ Significant at level of $5 \%$.

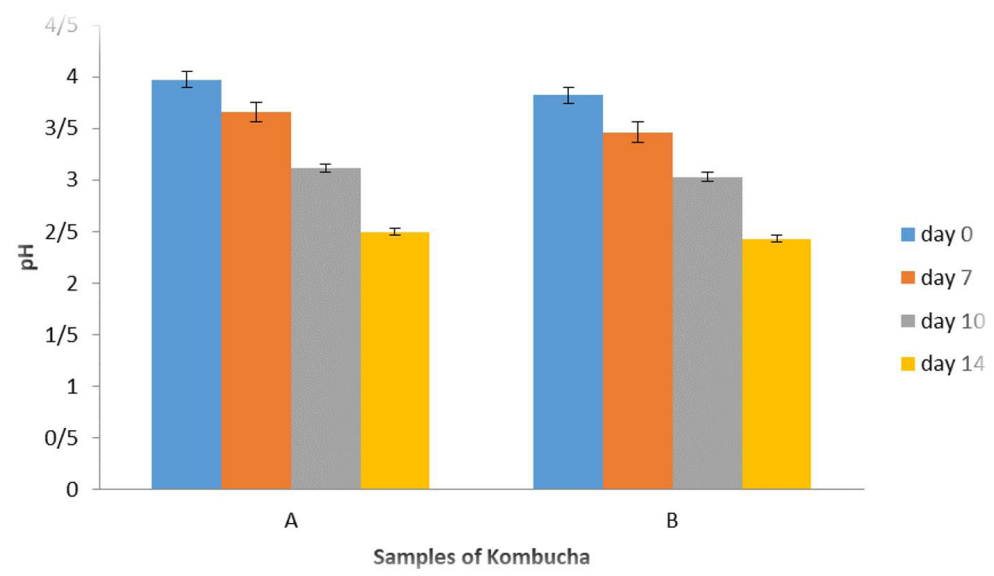

Figure 3. Comparison of $\mathrm{pH}$ changes in optimized sample of kombucha solutions; before drying (A) and after drying (B) on different days of fermentation. 

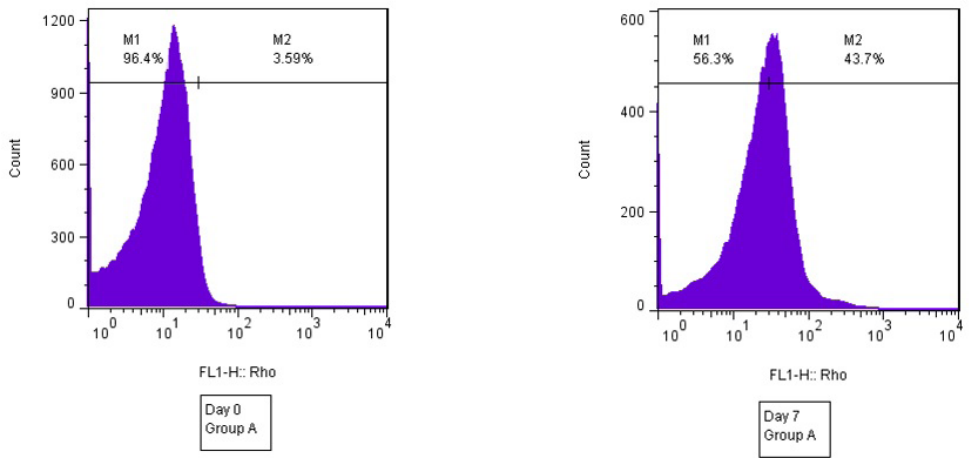

A
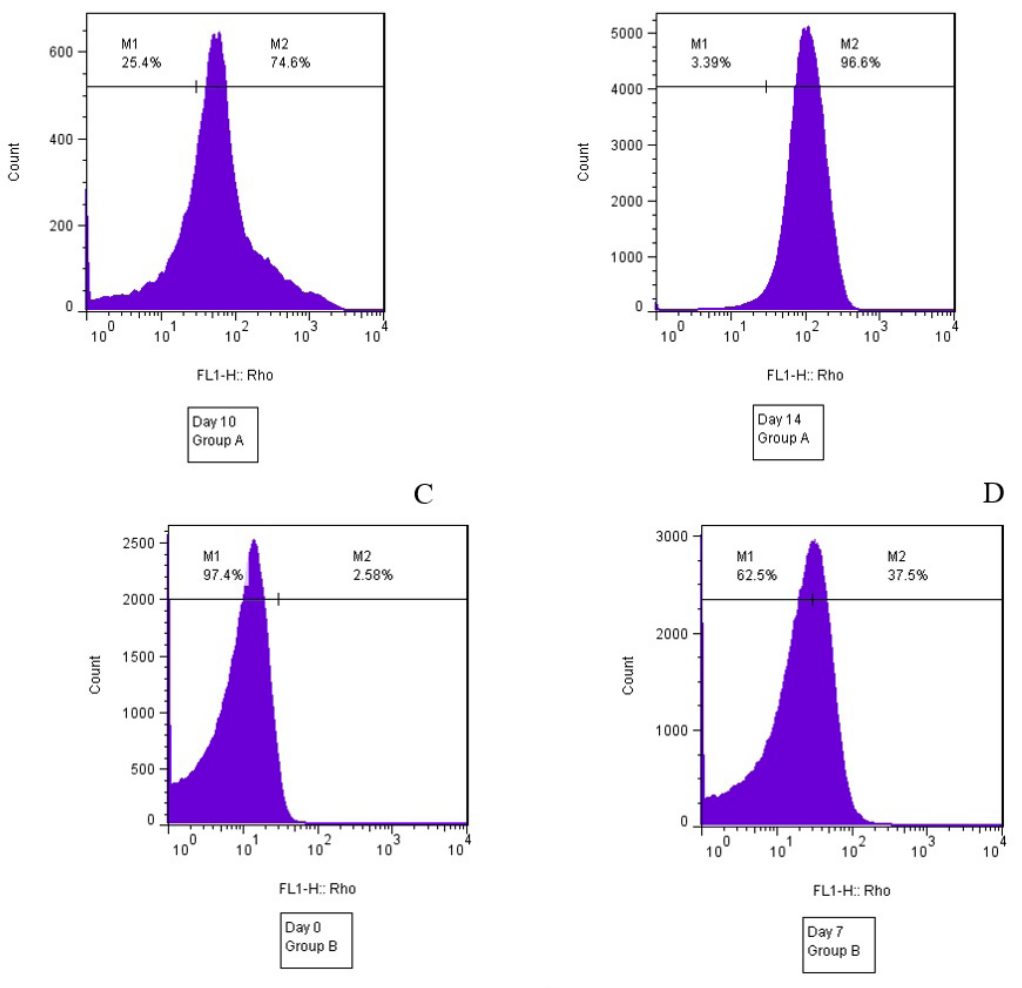

E
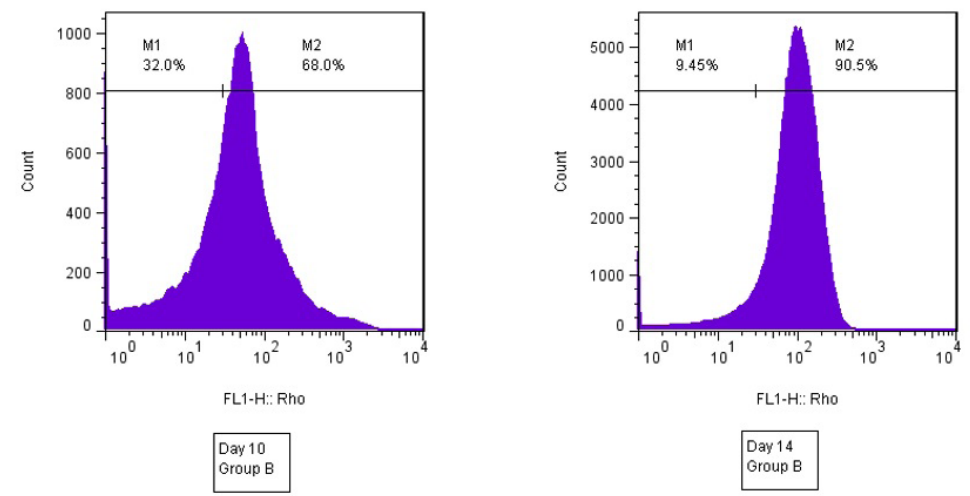

G

$\mathrm{H}$

Figure 4. Results for determining the viability of microorganisms using flow Cytometery (group A): (A) Results for day 0 (start of fermentation); (B) results for day 7; (C) results for day 10; (D) results for day 14. (group B): (E) Results for day 0 (start of fermentation); (F) results for day 7; (G) results for day $10 ;(\mathrm{H})$ results for day 14 . 
Table 3. Survival rate of microorganisms in kombucha solution before (A) and after drying (B) by fungi and bacteria.

\begin{tabular}{cccc}
\hline Samples & days & Viability of bacteria (\%) & Viability of fungi (\%) \\
\hline group A & 0 & 0.01 & 1.63 \\
& 7 & 11.69 & 46.48 \\
& 10 & 45.71 & 65.77 \\
& 14 & 95.28 & 78.50 \\
group B & 0 & 0.00 & 1.24 \\
& 7 & 8.66 & 41.28 \\
& 10 & 40.90 & 61.89 \\
& 14 & 90.01 & 73.19 \\
\hline
\end{tabular}

In both groups, the survival rate for both bacteria and fungi increased over the fermentation time.

\subsection{Results of sensory evaluation of kombucha drink}

In this study, kombucha drink was prepared in two groups of control (kombucha solution before drying) and experimental group (kombucha solution after drying). And in order to better and more accurately understanding of the nature of this drink, the opinion of 15 reputable evaluators on some factors such as odor, taste, color, appearance (clarity and turbidity), and overall acceptance, for both groups (control and experiment) in intervals Different times (at the beginning of the experiment, the day 7 , the day 10 , and 14 days after the start of the experiment) were recorded.

Comparison of the average opinions of the 15 evaluators in the studied variables in different time periods according to the experimental stage

Mean scores provided by the 15 evaluators in relation to the variables of odor, taste, color, appearance, and overall acceptance of Kombucha drink during the intervals of 0, 7, 10, and 14 days separately in the pre- and post-drying stages in Figure 5A-E are provided. Also, the consistency of the mean scores of four time periods $(0,7,10$, and 14 days) was examined by Friedman test. In addition, for the variables that include the asterisk, a test was performed to compare the mean pairs by Mann-Whitney test. The results of these tests are indicated using English letters in indexes 6 to 10. According to the contract, columns that have at least one common English letter have no statistically significant differences with each other. In Figure 5A, the mean scores of kombucha drink aroma in the pre-drying phase were statistically almost identical in all time periods from the beginning of the experiment to the end of the 14th day. However, in the postdrying phase of kombucha drink, the mean aroma scores at the beginning of the experiment were significantly lower than the later time period (7th, 10th, and 14th days). In Figure 5B the mean taste scores of kombucha drink in the pre- and post-drying stages on the 14th day were statistically different from the time intervals of 0 and the 7 th day of the experiment; But there was no significant difference with the 10th day. In Figure 5C, the mean color scores of Kombucha beverage in the pre-drying phase at all-time intervals from the beginning of the experiment to the end of the 14th day were not statistically significant. But in the post-drying phase of kombucha drink, the mean color scores at the beginning of the experiment were significantly lower on the 7th, 10th, and 14th days of the experiment. In Figure 5D, the mean scores of the kombucha drink appearance factor before drying from the beginning of the experiment to the end of the 14th day were statistically almost the same. However, average appraisal scores on the appearance of the kombucha drink in the post-drying phase on the 14th day were significantly higher than the time interval of 0 and 7 days, but there was no statistically significant difference with the 10th day. In Figure 5E, the mean scores of overall acceptance of kombucha drink in before drying stage were not statistically significantly different in all time periods from the beginning of the experiment to the end of the 14 day. However, in the post-drying stage of kombucha drink, the average total acceptance scores of the 15 evaluators at the beginning of the experiment were significantly lower than other time periods (seventh, tenth and fourteenth days of the experiment).

\section{Discussion}

In this study, the $\mathrm{pH}$ of kombucha had a range of 2.064. By increasing the fermentation time, the $\mathrm{pH}$ of samples was decreased due to conversion of sucrose to organic acids (Markov et al., 2001; Malbasa et al., 2011).pH value less than 4.2 during the first 7 days of fermentation, indicates unsuccessful fermentation (due to contamination or fermentation in inappropriate temperature). If $\mathrm{pH}$ value reach to less than 2.5 , beverage is not suitable from sensory aspects.

In this study, isolation and purification of microorganisms from kombucha beverage was performed using molecular and phylogenetic methods. Komagateobacter and Lactobacillus species were the most populous bacteria that isolated from kombucha solution in this study. The simultaneous and high presence of two species indicates the fact that most of the antioxidant activity of this drink is primarily due to the metabolic activity of both species, which has been shown in previous studies (Jayabalan et al., 2014; Markov et al., 2001; Kozyrovska et al., 2012). These key species increase the bioavailability of polyphenols and antioxidants in kombucha tea. Lactobacillus species, which are often present in the cellulose layer, have potential therapeutic properties and can act as a probiotic organism. Such microorganisms balance the microflora of the gastrointestinal tract, facilitate the movement of substances in the intestines, and promote immune system. Several mechanisms have been proposed for their preventive and therapeutic effects in human diseases, which can produce compounds. Bacterial inhibitors, modulating intestinal $\mathrm{pH}$, strengthening the immune system, blocking bacterial outlets, and competing for nutrient uptake were noted (Vanderpool et al., 2008). Due to the importance of lactic acid bacteria in terms of probiotic properties, the use of these bacteria in the food industry is increasing. Because different strains have several fermentation properties, such as acid production, they have flavor. Undoubtedly, the most important application of lactic acid bacteria is their use as a starter in the production of fermented foods.

In a study by Gaggia et al. (2018) on the microbiology and antioxidant activity of kombucha made from black, green, and rooibos tea, kumagateo bacter was the most abundant species isolated in black and green tea. The results of this study are consistent with 


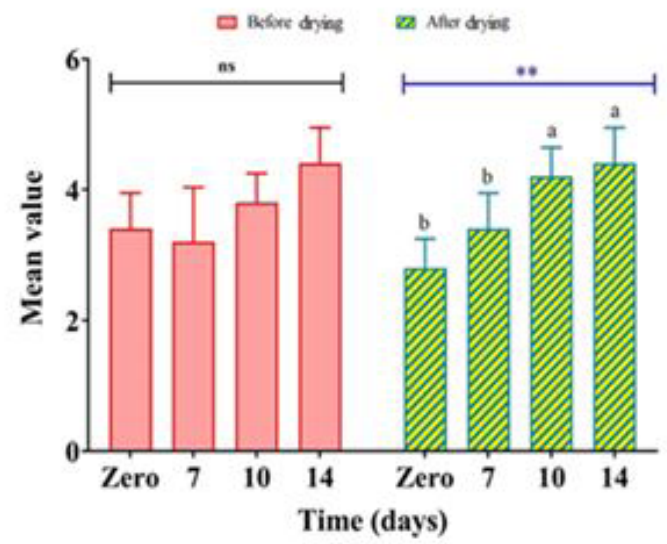

A. aroma during the time intervals

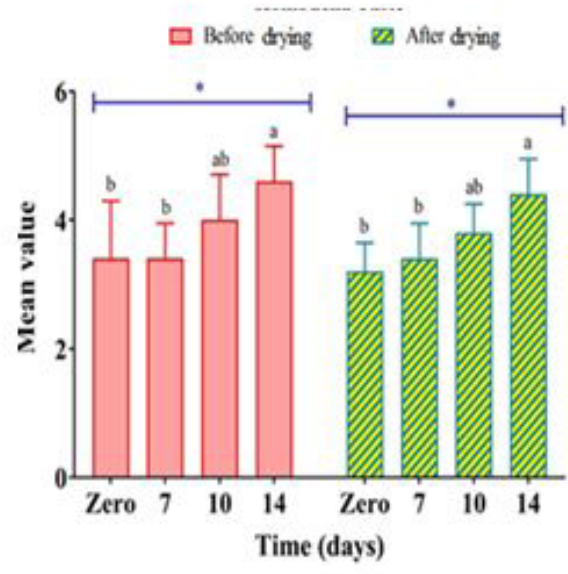

B. taste during the time intervals a adien ding a Mordyis

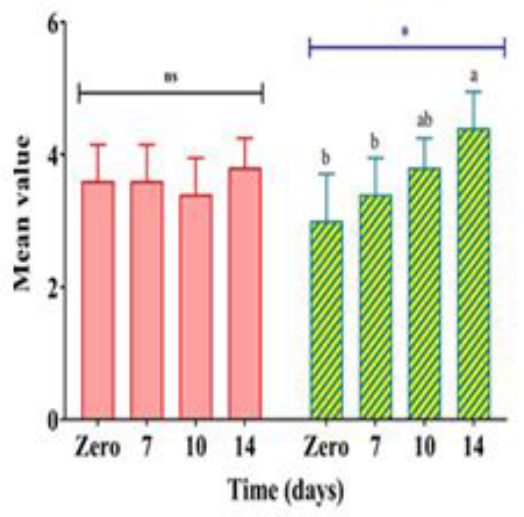

D. appearance during the time intervals

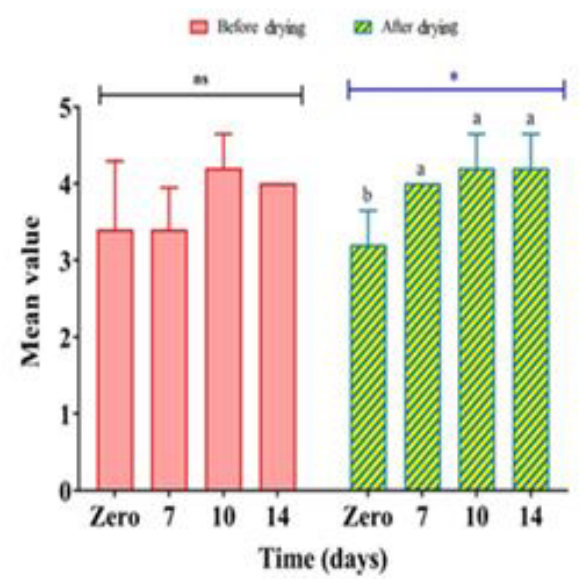

C. color during the time intervals

D Bowe ding a Marding

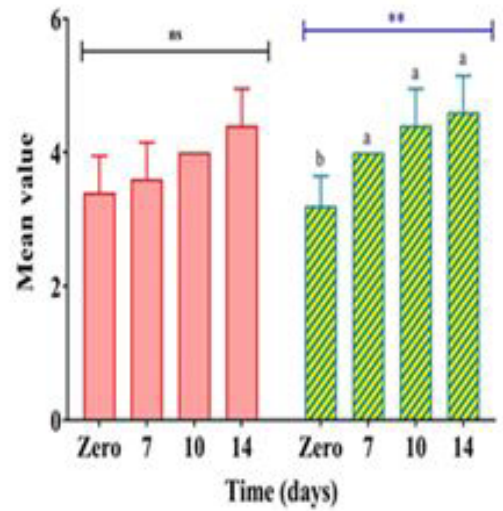

E. General acceptance during the time intervals

Figure 5. Characteristics of Kombucha drink before and after drying by time intervals, aroma during the time intervals (A), taste during the time intervals (B), color during the time intervals (C), appearance during the time intervals (D), aroma during the time intervals (E). Values are presented as mean \pm standard deviation for five replications. The results of these tests are indicated by the asterisk $\left(^{*}\right)$ in the second index. The mean values in the columns that have a common English letter are not statistically significantly different from each other $\left(\mathrm{p} \leq 0.01\left({ }^{* *}\right)\right.$. 
the present study in terms of the type and frequency of isolated microorganisms. In a study by Marsh et al. (2014) which analyzed bacteria and fungi isolated from kombucha solution on different days, Gluconobacter was the predominant bacterial species isolated in $85 \%$ of samples and Lactobacillus in abundance above $30 \%$, were identified as the second dominant isolated samples. Acetobacteria were present in only $2 \%$ of the isolated samples. The predominant isolated yeast was Zygosaccharomyces with a frequency of over $95 \%$. The results of this study are consistent with the present study in terms of the type of bacterial species isolated. The reason for the difference in the type of isolated samples is probably due to differences in geographical area. Teoh et al. (2004) reported that the coexistence of kombucha tea depends on the bacterial and yeast species, as well as the geographical conditions and local climate of each region.

Starmerella bacilaris was one of the yeasts that isolated in this study, which was isolated for the first time from kombucha solution. Starmerlla bacilaris is a non-saccharomyces ascomycete yeast found in spontaneous fermentations and has a relatively high population of about $10^{4}-10^{6}$ cells per mL. Starmerlla bacilaris is commonly found in biological environments and sometimes isolated from soil, fruit insects, and some rotten fruits (Lemos et al., 2017). The potential application of Starmerala bacilaris has been considered in wine making and in mixed fermentations with Saccharomyces cerevisiae because of its fructophilicity and ability to produce wine by reducing ethanol levels and increasing the organoleptic properties of wine (Englezos et al., 2016) and the possible uses of this yeast as an antifungal agent against the botrytis are also being studied (Lemos et al., 2016). Starmerlla bacilaris is an osmotolerant species (Sipiczki, 2003) and is one of the promising species to meet the needs of the fermentation market among non-saccharomyces yeasts. In particular, it produces less ethanol, and less biogenic amines and volatile acids than Saccharomyces cerevisiae (Lemos et al., 2016).

DPPH and CUPRAC methods were used to determine the antioxidant activity. Results showed that CUPRAC was accurate and efficient method could replace with other methods or in combination with the DPPH method. This method can measure antioxidants unspecified by other methods such as FRAP (which works with iron) (Apak et al., 2008). Results of the present study showed an increase in DPPH radical inhibition activity and antioxidant production by kombucha over the fermentation time. Aloulou et al. also showed that in kombucha fermentation phenol content, antioxidant activity, and inhibitory activity of alpha-amylase enzyme commonly consumed in five teas (Aloulou et al., 2012). Velićanski et al. (2014) showed that in a fermented solution of lemon balm increased phenolic compounds (increase 1.3, 1.9 and 4.6 times, respectively) as well as antioxidant activity against DPPH radicals. Fu et al. (2014) indicates that green tea had the greatest DPPH free radical removal activity among three types of tea. Gaggia et al. (2018) showed the antioxidant activity of various kombucha drink made from green, black and rooibos tea.

The sensory properties of kombucha solution were investigated by 15 trained evaluators. The five senses were considered including sight, hearing, touch, smell, and taste. The visual (product appearance), the olfactory (aroma profile), the taste (sweet, sour) and the senses (e.g., through the chemical sensation of the tongue tingling with carbon dioxide bubbles) have been studied. There is a correlation between the chemical composition of kombucha and sensory properties (Tran et al., 2020). Monosaccharides glucose and fructose have a sweetness of $65-75 \%$ and $120 \%(w / w)$, respectively, compared to sucrose (Villarreal-Soto et al., 2018; Jayabalan et al., 2014). Polyphenols, $\mathrm{CO}_{2}$ and ethanol play main role in the oral sensation of the product. Before any treatment, 30 to $40 \%$ of the dried weight of tea consists of polyphenols. Origin of bitter taste of kombucha is caffeine and polyphenols of tea leaves which should be covered by sweetness, (Chakravorty et al., 2016). Also organic acids, mainly produced by acetic acid bacteria, are the main reason of its sour taste. Yeasts and lactic acid bacteria are also involved to some extent in making kombucha flavor (Blanc, 1996; Chakravorty et al., 2016; Jayabalan et al., 2007). The smell of kombucha, comes from both the main substrate (tea) and also the volatile metabolites (2-phenyl ethanol, linalool, 3-hexenol, geraniol and acetic acid) produced by microorganisms (Rosend et al., 2019). Turbidity, is due to the colloidal state which is the results of presence of microorganisms and the accumulation of polyphenols, proteins, and fibers produced by acetic acid bacteria (Zhang et al., 2018; Jayabalan et al., 2007).

Polyphenolic pigments (e.g. theaflavin, as a -reddishorange) extracted from tea leaves is responsible for the color of kombucha. During the kombucha fermentation decreased color intensity and increased in total phenolic content are reported (Chakravorty et al., 2016; Chu \& Chen, 2006). Decreased pH, and biological activity in some cases changes or even destroys the pigments extracted from tea (Haslam, 2003). Neffe-Skocińska et al. (2017), studied on sensory analysis of kombucha drinks prepared from green and black tea by 16 trained evaluators for 10 days at 20,25 and $30{ }^{\circ} \mathrm{C}$ (Neffe-Skocińska et al., 2017). More research seems required for the sensory properties of kombucha.

Rapid assessment of the presence of microbes and their survival is extremely important for food products. Interest in developing more efficient methods in industrial microbiology has increased in recent decades. The flow cytometric method satisfies these requirements and provides an application for determining the total number of microorganisms in beverages and foods (Malacrinò et al., 2001). According to present study, the viability of microorganisms was investigated using flow cytometery. For microorganisms in particular, viability is a term that is difficult to define and therefore difficult to measure. A traditional way to determine survival of microorganisms is to examine the culture (i.e., ability to multiply); But determining cultivability is often very slow. Flow cytometery allows us to check a large number of cells in terms of viability by quick and quantitative dye absorption measurement. Rhodamine 123 dye is a cationic dye that penetrates into living bacteria and accumulates in cells due to the negative charge on the inner surface of the membrane. In this case, dye-accumulated living cells have an absorption in the range of $525 \mathrm{~nm}$ and their percentage can be determined using flow cytometery (Gholami \& Etemadifar, 2013). In the study of kombucha, the viability of microorganisms has been investigated using the CFU method (Watawana et al., 2018; Goh et al., 2012; Yikmis \& Tuggum, 2019; Coton et al., 2017). This present study used flow cytometery for the first time to determine the survival rate of microorganisms in Kombucha solution. Due to the fact that the beneficial properties of kombucha drink largely depend on the survival and the presence of microorganisms in the kombucha solution and also metabolites produced by them. Determining the survival rate of bacteria and yeasts in kombucha drink using flow cytometery is much faster, more sensitive, and more accurate 
than cultivation methods. The results of flow cytometery showed increased survival of microorganisms during the fermentation, and this indicates an increase in the adaptation of microorganisms to the environment and conditions of fermentation over time and their ability to use the substrate and increase their number and durability in kombucha solution.

The results of the present study are consistent with the findings of other researchers in this field in terms of increasing the population of microorganisms over the fermentation time (Watawana et al., 2018) and differs from the results of some studies due to the reduced population of microorganisms during the fermentation (Yikmis \& Tuggum, 2019; Coton et al., 2017). Because the culture of kombucha does not have the same microbiological standard from different geographical areas. Therefore, the results of microbial analysis are normal (Teoh et al., 2004).

\section{Conclusion}

Given the changes in human lifestyle in today's world and the increase in diseases caused by oxidative stress, the simplest and most effective way is to include foods containing antioxidants in the daily diet. Kombucha is a useful fermented beverage rich in antioxidants such as polyphenols and flavonoids. Probiotic and prebiotic compounds play a vital role in human health. Popularity of kombucha is growing as a probiotic and prebiotic drink. Because determining the type of microbial population in fermented beverages is very important; In the present study, isolation and purification of microorganisms from kombucha solution was performed using culture method and molecular identification of microorganisms using PCR. Starmella bacillaris, which were isolated from kombucha for the first time in this study and Hanseniaspora uvarum are promising for the needs of the fermentation market among the non-Saccharomyces yeasts due to the lower ethanol production and lower levels of biogenic amines and volatile acids than Saccharomyces cerevisiae. Determining the survival rate of microorganisms in fermented beverages is an important principle for maintaining its nutritional value and beneficial properties. Flow cytometery is an accurate and rapid method for determining the survival rate of microorganisms, which in this study replaced the traditional method. According to the results obtained in this study, preparation of kombucha in powder form is a useful and costeffective method to maintain and maintain its beneficial properties. Further research on the sensory properties of kombucha can lead to a better definition of the quality of kombucha and better control of its production process.

\section{Acknowledgements}

The authors acknowledge financial support of Shahid Beheshti University of Medical Sciences for grant number 25446.

\section{References}

Aloulou, A., Hamden, K., Elloumi, D., Ali, M. B., Hargafi, K., Jaouadi, B., Ayadi, F., Elfeki, A., \& Ammar, E. (2012). Hypoglycemic and antilipidemic properties of Kombucha tea in alloxan-induced diabetic rats. BMC Complementary and Alternative Medicine Journal, 12, 63. http://dx.doi.org/10.1186/1472-6882-12-63. PMid:22591682.
Amarasinghe, H., Weerakkody, N. S., \& Waisundara, V. Y. (2018). Evaluation of physicochemical properties and antioxidant activities of Kombucha "Tea Fungus" during extended period of fermentation. Food Sciences \& Nutrition Journal, 6(3), 659-665. http://dx.doi. org/10.1002/fsn3.605. PMid:29876117.

Annuzzi, G., Bozzett, L., Costabile, G., Giacco, R., Mangione, A., Anniballi, G., Vitale, M., Vetrani, C., Cipriano, P., Della Corte, G., Pasanisi, F., Riccardi, G., \& Rivellese, A. A. (2014). Diets naturally rich in polyphenols improve fasting \& postprandial dyslipidemia and reduce oxidative stress: a randomized controlled trial. Journal of Clinical Nutrition, 99(3), 463-471. http://dx.doi.org/10.3945/ ajcn.113.073445. PMid:24368433.

Apak, R., Guclu, K., Ozyurek, M., \& Çelik, S. E. (2008). Mechanism of antioxidant capacity assays and the CUPRAC (cupric ion reducing antioxidant capacity) assay. Mikrochimica Acta, 160(4), 413-419. http://dx.doi.org/10.1007/s00604-007-0777-0.

Battikh, H., Chaieb, K., Bakhrouf, A., \& Ammar, E. (2013). Antibacterial and antifungal activities of black and green Kombucha teas. Journal of Food Biochemistry, 37(2), 231-236. http://dx.doi.org/10.1111/j.17454514.2011.00629.x.

Blanc, P. J. (1996). Characterization of the tea fungus metabolites. Biotechnology Letters, 8(2), 139-142. http://dx.doi.org/10.1007/ BF00128667.

Chakravorty, S., Bhattacharya, S., Chatzinotas, A., Chakraborty, W., Bhattacharya, D., \& Gachhui, R. (2016). Kombucha tea fermentation: microbial and biochemical dynamics. International Journal of Food Microbiology, 220, 63-72. http://dx.doi.org/10.1016/j. ijfoodmicro.2015.12.015. PMid:26796581.

Chu, S. C., \& Chen, C. (2006). Effects of origins and fermentation time on the antioxidant activities of Kombucha. Food Chemistry, 98(3), 502-507. http://dx.doi.org/10.1016/j.foodchem.2005.05.080.

Coton, M., Pawtowski, A., Taminiau, B., Burgaud, G., Deniel, F., Coulloumme-Labarthe, L., Fall, A., Daube, G., \& Coton, E. (2017). Unraveling microbial ecology of industrial-scale Kombucha fermentations by metabarcoding and culture-based methods. FEMS Microbiology Ecology, 93(5), 1-16. http://dx.doi.org/10.1093/femsec/ fix048. PMid:28430940.

Dufresne, C., \& Farnworth, E. (2000). Tea kombucha, and health: a review. Food Research, 33(6), 409-421. http://dx.doi.org/10.1016/ S0963-9969(00)00067-3.

Englezos, V., Rantsiou, K., Cravero, F., Torchio, F., Ortiz-Julien, A., Gerbi, V., Rolle, L., \& Cocolin, L. (2016). Starmerella bacillaris and Saccharomyces cerevisiae mixed fermentations to reduce ethanol content in wine. Applied Microbiology and Biotechnology, 100(12), 5515-5526. http://dx.doi.org/10.1007/s00253-016-7413-z. PMid:26960321.

Fu, C., Yan, F., Cao, Z., Xie, F., \& Lin, J. (2014). Antioxidant activities of Kombucha prepared from three different substrates and changes in content of probiotics during storage. Food Science and Technology, 34(1), 123-126. http://dx.doi.org/10.1590/S010120612014005000012.

Gaggia, F., Baffoni, L., Galiano, M., Nielsen, D. S., Jakobsen, R. R., Castro-Mejía, J. L., Bosi, S., Truzzi, F., Musumeci, F., Dinelli, G., \& Di Gioia, D. (2018). Kombucha beverage from green, black and rooibos teas: a comparative study looking at microbiology, chemistry and antioxidant activity. Nutrients, 11(1), 1-22. http://dx.doi.org/10.3390/ nu11010001. PMid:30577416.

Gamboa-Gómez, C. I., González-Laredo, R. F., Gallegos-Infante, J. A., Pérez, M. D., Moreno-Jiménez, M. R., Flores-Rueda, A. G., \& RochaGuzmán, N. E. (2016). Antioxidant and angiotensin-converting enzyme inhibitory activity of Eucalyptus camaldulensis and Litsea 
glaucescens infusions fermented with Kombucha consortium. Food Technology and Biotechnology, 54(3), 367-374. http://dx.doi. org/10.17113/ftb.54.03.16.4622. PMid:27956869.

Gholami, M., \& Etemadifar, Z. (2013). Isolation and molecular identification of a new strain of Microbacterium resistens, capable of tolerating the extreme conditions. Biological Journal of Microorganism, 2(5), 27-42.

Goh, W. N., Rosma, A., Kaur, B., Fazilah, A., Karim, A. A., \& Bhat, R. (2012). Fermentation of black tea broth (Kombucha): I. effects of sucrose concentration and fermentation time on the yield of microbial cellulose. International Food Research Journal, 19(1), 109-117.

Haslam, E. (2003). Thoughts on thearubigins. Phytochemistry, 64(1), 61-73. http://dx.doi.org/10.1016/S0031-9422(03)00355-8. PMid:12946406.

Jayabalan, R., Malbasa, R. V., Loncar, E. S., Vitas, J. S., \& Sathishkumar, M. (2014). A review on kombucha tea: microbiology, composition, fermentation, beneficial effects, toxicity, and tea fungus. Comprehensive Reviews in Food Science and Food Safety, 13(4), 538-550. http:// dx.doi.org/10.1111/1541-4337.12073. PMid:33412713.

Jafari, R., Naghavi, N. S., Khosravi-Darani, K., Doudi, M., \& Shahanipour, K. (2020). Kombucha microbial starter with enhanced production of antioxidant compounds and invertase. Biocatalysis and Agricultural Biotechnology, 29, 101789. http://dx.doi.org/10.1016/j.bcab.2020.101789.

Jayabalan, R., Marimuthu, S., \& Swaminathan, K. (2007). Changes in content of organic acids and tea polyphenols during kombucha tea fermentation. Food Chemistry, 102(1), 392-398. http://dx.doi. org/10.1016/j.foodchem.2006.05.032.

Kozyrovska, N. O., Reva, O. M., Goginyan, V. B., \& De Vera, J. P. (2012). Kombucha microbiome as a probiotic: a view from the perspective of post-genomics and synthetic ecology. Biopolymers and Cell, 28(2), 103-113. http://dx.doi.org/10.7124/bc.000034.

Lemos, W. J. Jr., Bovo, B., Nadai, C., Crosato, G., Carlot, M., Favaron, F., Giacomini, A., \& Corich, V. (2016). Biocontrol ability and action mechanism of Starmerella bacillaris (synonym Candida zemplinina) isolated from wine musts against gray mold disease agent Botrytis cinerea on grape and their effects on alcoholic fermentation. Frontiers in Microbiology, 7, 1249. http://dx.doi.org/10.3389/fmicb.2016.01249. PMid:27574517.

Lemos, W. J. Jr., Treu, L., Duarte, V. D. S., Campanaro, S., Nadai, C., Giacomini, A., \& Corich, V. (2017). Draft genome sequence of the yeast Starmerella bacillaris (syn., Candida zemplinina) FRI751 isolated from fermenting must of dried Raboso grapes. Genome Announcements, 5(17), e00224-17. http://dx.doi.org/10.1128/ genomeA.00224-17. PMid:28450507.

Loncar, E., Djuric, M., Malbasa, R., Kolarov, L. J., \& Klasnja, M. (2006). Influence of working conditions upon kombucha conducted fermentation of black tea. Food and Bioproducts Processing, 84(3), 186-192. http://dx.doi.org/10.1205/fbp.04306.

Malacrinò, P., Zapparoli, G., Torriani, S., \& Dellaglio, F. (2001). Rapid detection of viable yeasts and bacteria in wine by flow cytometry. Journal of Microbiological Methods, 45(2), 127-134. http://dx.doi. org/10.1016/S0167-7012(01)00243-3. PMid:11311398.

Malbasa, R., Loncar, E. S., Vitas, J. S., \& Canadanovic-Brunet, J. M. (2011). Influence of starter cultures on the antioxidant activity of Kombucha beverage. Food Chemistry, 127(4), 1727-1731. http:// dx.doi.org/10.1016/j.foodchem.2011.02.048.

Malbasa, R., Vitas, J., Loncar, E., Grahovac, J., \& Milanovic, S. (2014). Optimisation of the antioxidant activity of Kombucha fermented milk products. Czech Journal of Food Sciences, 32(5), 477-484. http:// dx.doi.org/10.17221/447/2013-CJFS.
Markov, S., Malbasa, R., Hauk, M., \& Cvetkovic, D. (2001). Investigation of tea fungus microbe assotiations the yeast. Acta Periodica Technologica, 32, 133-138.

Marsh, A. J., O’Sullivan, O., Hill, C., Ross, R. P., \& Cotter, P. D. (2014). Sequence-based analysis of the bacterial and fungal compositions of multiple Kombucha (tea fungus) samples. Food Microbiology, 38, 171-178. http://dx.doi.org/10.1016/j.fm.2013.09.003. PMid:24290641.

Matei, B., Diguta, C. F., Popa, O., Cornea, C. P., \& Matei, F. (2018). Molecular identification of yeast isolated from different kombucha sources. The Annals of the University Dunarea de Jos of Galati. Fascicle VI, Food Technology, 42(1), 17-25.

Neffe-Skocińska, K., Sionek, B., Ścibisz, I., \& Kołożyn-Krajewska, D. (2017). Acid content and the effect of fermentation condition of kombucha tea beverage on physiochemical, microbiological and sensory properties. CYTA: Journal of Food, 15(4), 601-607. http:// dx.doi.org/10.1080/19476337.2017.1321588.

Reiss, J. (1994). Influence of different sugars on the metabolism of the tea fungus. Zeitschrift fur Lebensmittel-Untersuchung und -Forschung, 198(3), 258-261. http://dx.doi.org/10.1007/BF01192606.

Rosend, J., Kuldjarv, R., Rosenvald, S., \& Paalme, T. (2019). The effects of apple variety, ripening stage, and yeast strain on the volatile composition of apple cider. Heliyon, 5(6), e01953. http://dx.doi. org/10.1016/j.heliyon.2019.e01953. PMid:31211267.

Sanger, F., Nicklen, S., \& Coulson, A. R. (1977). DNA sequencing with chain-terminating inhibitors. Proceedings of the National Academy of Sciences of the United States of America, 74(12), 54635467. http://dx.doi.org/10.1073/pnas.74.12.5463. PMid:271968.

Shahbazi, H., Hashemi Gahruie, H., Golmakani, M.-T., Eskandari, M. H., \& Movahedi, M. (2018). Effect of medicinal plant type and concentration on physicochemical, antioxidant, antimicrobial, and sensorial properties of kombucha. Food Science \& Nutrition, 6(8), 2568-2577. http://dx.doi.org/10.1002/ fsn3.873. PMid:30510759.

Singleton, V., Orthofer, R., \& Lamuela-Raventós, R. (1999). Analysis of total phenols and other oxidation substrates and antioxidants by means of Fo-253. Czech Journal of Food Sciences, 4, 242-253.

Sipiczki, M. (2003). Candida zemplinina sp. nov., an osmotolerant and psychrotolerant yeast that ferments sweet botrytized wines. International Journal Of Systematic and Evolutionary Microbiology, 53(Pt 6), 2079-2083. http://dx.doi.org/10.1099/ijs.0.02649-0. PMid:14657149.

Sreeramulu, G., Zhu, Y., \& Knol, W. (2000). Kombucha fermentation and its antimicrobial activity. Journal of Agricultural and Food Chemistry, 48(6), 2589-2594. http://dx.doi.org/10.1021/jf991333m. PMid:10888589.

Srihari, T., \& Satyanarayana, U. (2012). Changes in free radical scavenging activity of kombucha during fermentation. Journal of Pharmaceutical Sciences \& Research, 4(11), 1978-1981.

Teoh, A. L., Heard, G., \& Cox, J. (2004). Yeast ecology of Kombucha fermentation. International Journal of Food Microbiology, 95(2), 119-126. http://dx.doi.org/10.1016/j.ijfoodmicro.2003.12.020. PMid:15282124.

Tran, T., Grandvalet, C., Verdier, F., Martin, A., Alexandre, H., \& TourdotMarechal, R. (2020). Microbiological and technological parameters impacting the chemical composition and sensory quality of kombucha. Comprehensive Reviews in Food Science and Food Safety, 19(4), 20502070. http://dx.doi.org/10.1111/1541-4337.12574. PMid:33337078.

Vanderpool, C., Yan, F., \& Polk, D. B. (2008). Mechanisms of probiotic action: implications for therapeutic applications in inflammatory bowel diseases. Inflammatory Bowel Diseases, 
14(11), 1585-1596. http://dx.doi.org/10.1002/ibd.20525. PMid:18623173.

Vazquez-Cabral, B. D., Rocha-Guzman, N. E., Gallegos-Infante, J. A., Gonzalez-Herrera, S. M., Gonzalez-Laredo, R. F., Moreno-Jimenez, M. R., \& Cordova-Moreno, I. T. S. (2014). Chemical and sensory evaluation of a functional beverage obtained from infusions of oak leaves (Quercus resinosa) inoculated with the Kombucha consortium under different processing conditions. Nutrafoods Journal, 13(4), 169-178. http://dx.doi.org/10.1007/s13749-014-0035-0.

Velićanski, A. S., Cvetković, D. D., Tumbas Šaponjac, V. T., \& Vulić, J. J. (2014). Antioxidant and antibacterial activity of the beverage obtained by fermentation of sweetened lemon balm (Melissa officinalis L.) tea with symbiotic consortium of bacteria and yeasts. Food Technology and Biotechnology, 52(4), 420-429. http://dx.doi. org/10.17113/ftb.52.04.14.3611. PMid:27904315.

Villarreal-Soto, S. A., Beaufort, S., Bouajila, J., Souchard, J. P., \& Taillandier, P. (2018). Understanding Kombucha tea fermentation: a review. Journal of Food Science, 83(3), 580-588. http://dx.doi.org/10.1111/17503841.14068. PMid:29508944.
Watawana, M. I., Jayawardena, N., \& Waisundara, V. Y. (2018). Valueadded Tea (Camellia sinesis) as a Functional Food using the Kombucha 'Tea Fungus.' Warasan Khana Witthayasat Maha Witthayalai Chiang Mai, 45(1), 136-146.

Yikmis, S., \& Tuggum, S. (2019). Evaluation of microbiological, physicochemical and sensorial properties of purple Basill Kombucha beverage. Turkish Journal of Agriculture Food Science and Technology, 7(9), 1321-1327. http://dx.doi.org/10.24925/ turjaf.v7i9.1321-1327.2550.

Zhang, W., Wang, X., Qi, X., Ren, L., \& Qiang, T. (2018). Isolation and identification of a bacterial cellulose synthesizing strain from kombucha in different conditions: Gluconacetobacter xylinus ZHCJ618. Food Science and Biotechnology, 27(3), 705-713. http:// dx.doi.org/10.1007/s10068-018-0303-7. PMid:30263796.

Zhao, Z., Sul, Y., Wu, H., Zhou, C., Hu, X., \& Zhang, J. (2018). Flavor chemical dynamics during fermentation of Kombucha tea. Emirates Journal of Food and Agriculture, 30, 732-741. http://dx.doi.org/10.9755/ ejfa.2018.v30.i9.1794. 
Appendix A. phylogenetic tree for the isolated specimens.
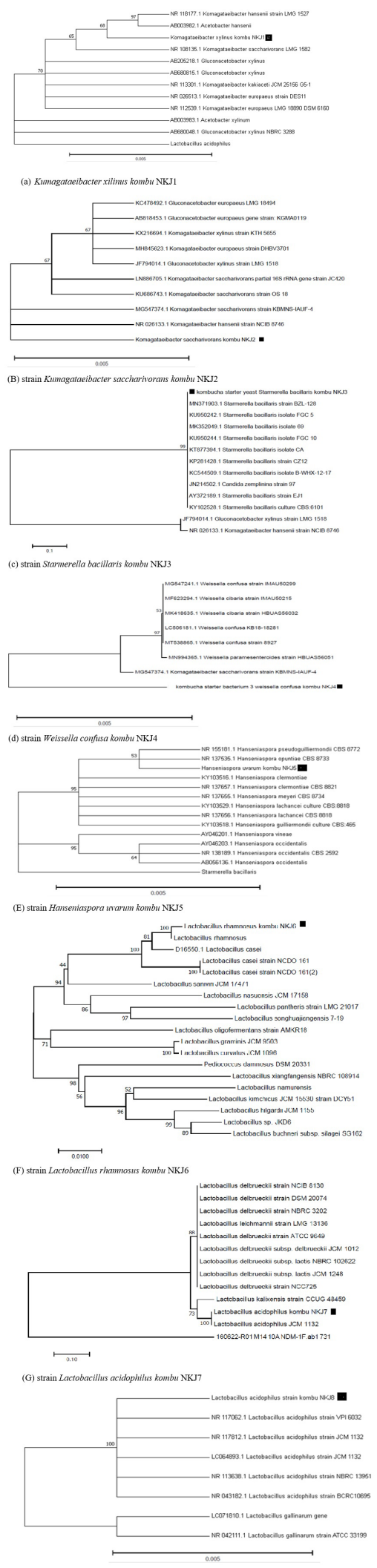

Figure A1. Phylogenetic tree of strain Kumagataeibacter xilinus kombu NKJ1 (a), strain Kumagataeibacter saccharivorans kombu NKJ2 (b), strain Starmerella bacillary kombu NKJ3 (c), strain Weissella confusa kombu NKJ4 (d), strain Hanseniaspora uvarum kombu NKJ5 (e), strain Lactobacillus rhamnosus kombu NKJ6 (f), strain Lactobacillus acidophilus kombu NKJ7 (g), strain Lactobacillus acidophilus kombu NKJ8 (h).

*The scale of the phylogenetic tree indicates the number of nucleotide substitutions at each compared site 